\title{
Didymin reverses phthalate ester-associated breast cancer aggravation in the breast cancer tumor microenvironment
}

\author{
YA-LING HSU $^{1 *}$, CHIA-JUNG HSIEH ${ }^{1,2 *}$, EING-MEI TSAI ${ }^{1}$, JEN-YU HUNG $^{3,4}$, \\ WEI-AN CHANG ${ }^{4,5}$, MING-FENG HOU ${ }^{5}$ and PO-LIN KUO ${ }^{5-7}$
}

\footnotetext{
${ }^{1}$ Graduate Institute of Medicine, College of Medicine, Kaohsiung Medical University; ${ }^{2}$ Department of Chinese Medicine, Kaohsiung Medical University Hospital; ${ }^{3}$ School of Medicine, College of Medicine, Kaohsiung Medical University; ${ }^{4}$ Division of Pulmonary and Critical Care Medicine, Kaohsiung Medical University Hospital; ${ }^{5}$ Institute of Clinical Medicine, College of Medicine; ${ }^{6}$ Research Center for Environmental Medicine, Kaohsiung Medical University, Kaohsiung 807; ${ }^{7}$ Institute of Medical Science and Technology, National Sun Yat-Sen University, Kaohsiung 80424, Taiwan, R.O.C.
}

Received January 22, 2015; Accepted November 18, 2015

DOI: $10.3892 / \mathrm{ol} .2015 .4008$

\begin{abstract}
The present study demonstrated two novel findings. To the best of our knowledge, it is the first study to demonstrate that regulated upon activation, normal T-cell expressed and secreted (RANTES), produced by breast tumor-associated monocyte-derived dendritic cells (TADCs) following breast cancer cell exposure to phthalate esters, may contribute to the progression of cancer via enhancement of cancer cell proliferation, migration and invasion. Furthermore, the present study revealed that didymin, a dietary flavonoid glycoside present in citrus fruits, was able to reverse phthalate ester-mediated breast cancer aggravation. MDA-MB-231 cells were treated with butyl benzyl phthalate (BBP), di- $n$-butyl phthalate (DBP) or di-2-ethylhexyl phthalate (DEHP). Subsequently, the conditioned medium (CM) was harvested and cultured with monocyte-derived dendritic cells (mdDCs). Cultures of MDA-MB-231 cells with the conditioned medium of BBP-, DBP- or DEHP-MDA-MB-231 tumor-associated mdDCs (BBP-, DBP- or DEHP-MDA-TADC-CM) demonstrated enhanced proliferation, migration and invasion. Exposure of the MDA-MB-231 cells to DBP induced the MDA-TADCs to produce the inflammatory cytokine RANTES, which subsequently induced MDA-MB-231 cell proliferation, migration and invasion. Depleting RANTES reversed the effects of DBP-MDA-TADC-mediated MDA-MB-231 cell proliferation,
\end{abstract}

Correspondence to: Professor Ming-Feng Hou or Professor Po-Lin Kuo, Institute of Clinical Medicine, College of Medicine, Kaohsiung Medical University, 100 Shih-Chuan 1st Road, Kaohsiung 807, Taiwan, R.O.C.

E-mail: mifeho@kmu.edu.tw

E-mail: kuopolin@seed.net.tw

*Contributed equally

Key words: phthalate esters, breast cancer, tumor microenvironment, didymin migration and invasion. In addition, didymin was observed to suppress phthalate-mediated breast cancer cell proliferation, migration and invasion. The present study suggested that didymin was capable of preventing phthalate ester-associated cancer aggravation.

\section{Introduction}

Phthalates, including butyl benzyl phthalate (BBP), di- $n$-butyl phthalate (DBP) and di-2-ethylhexyl phthalate (DEHP), are utilized as softeners and plasticizers $(1,2)$. Epidemiological studies have observed that phthalate exposure may increase the risk of breast cancer (3-6). However, the effects of phthalate esters in the breast cancer tumor microenvironment remain to be elucidated.

The tumor microenvironment is known to have a significant role in the progression of tumors and the development of chemoresistance to anticancer drugs (7). The tumor microenvironment is comprised of stromal cells, immune cells [lymphocytes, macrophages and dendritic cells (DCs)], growth factors, extracellular matrix constituents, metabolites and cytokines/chemokines (8). As antigen-presenting cells, DCs have been observed to exhibit significant roles in the initiation and regulation of the immune response to cancer (9). Tumor-associated DCs (TADCs) have been observed to contribute to the metastasis of tumors in various cancers $(10,11)$. Regulated upon activation, normal T-cell expressed and secreted (RANTES), also known as $\mathrm{C}-\mathrm{C}$ chemokine ligand 5 , is a cytokine consistently observed in increased levels in breast cancer subtypes (12), and has been observed to be associated with the progression of breast cancer and the promotion of metastasis (13-16).

Epidemiological studies have provided evidence that a high dietary intake of flavonoids via fruits and vegetables may be associated with reduced cancer rates in humans (17-20). Flavonoids are a class of phenolic compounds that are widely distributed throughout the plant kingdom; they display diverse biological activities, including the inhibition of tumor progression and the prevention of cancer initiation $(21,22)$. Didymin, a dietary flavonoid glycoside present in citrus fruits, demonstrates antioxidant and anticancer properties (23-28). 
The present study evaluated the effects of phthalate esters in the breast cancer tumor microenvironment and investigated didymin, a dietary flavonoid glycoside present in citrus fruits, as a possible antidote for phthalate ester-associated cancer aggravation.

\section{Materials and methods}

Chemicals. Didymin was obtained from Extrasynthese (Genay, France), and was dissolved in dimethyl sulfoxide (DMSO; Sigma-Aldrich, St. Louis, MO, USA) and stored at $-20^{\circ} \mathrm{C}$. Control cultures received the carrier solvent $(0.1 \%$ DMSO). All other chemicals utilized were in the purest form available commercially.

Cell culture and conditioned medium. Human breast adenocarcinoma MDA-MB-231 cells (American Type Culture Collection, Manassas, VA, USA) were cultured in $\alpha$-minimum essential medium ( $\alpha$-MEM; Thermo Fisher Scientific, Inc., Waltham, MA, USA) supplemented with non-essential amino acids, $0.1 \mathrm{mmol} / 1$ sodium pyruvate, $1 \%$ antibiotic/anti-mycotic solution and $10 \%$ fetal bovine serum (FBS) (all Thermo Fisher Scientific, Inc.). In order to obtain the various conditioned media (CM), the MDA-MB-231 cells $\left(2 \times 10^{6} / 100-\mathrm{mm}\right.$ dish) were treated with or without BBP, DBP or DEHP (all Sigma-Aldrich) at identical concentrations of $1 \mu \mathrm{M}$ for $6 \mathrm{~h}$. Following washing and culturing for $24 \mathrm{~h}$, the CM of phthalate ester-treated MDA-MB-231 cells (BBP-, DBP- or DEHP-MDA-CM) were harvested (Fig. 1A).

Isolation of cluster of differentiation $(C D) 14^{+}$monocytes and differentiation of monocyte-derived dendritic cells (mdDCs). Monocytes were purified from peripheral blood mononuclear cells obtained from healthy consenting donors. Mononuclear cells were isolated from the blood by Ficoll-Hypaque gradient (GE Healthcare Life Sciences, Chalfont, UK). $\mathrm{CD}_{14}{ }^{+}$monocytes were purified with $\mathrm{CD} 14^{+}$monoclonal antibody-conjugated magnetic beads (MACS MicroBeads; Miltenyi Biotec GmbH, Bergisch Gladbach, Germany), according to the manufacturer's protocols. mdDCs were generated by culturing $\mathrm{CD} 14^{+}$monocytes in $\alpha$-MEM containing FBS and $20 \mathrm{ng} / \mathrm{ml}$ granulocyte-macrophage colony-stimulating factor (GM-CSF) and $10 \mathrm{ng} / \mathrm{ml}$ interleukin (IL)4 (R\&D Systems, Inc., Minneapolis, MN, USA) for 5 days. The medium was replaced with fresh medium containing GM-CSF and IL4 on day 3. For the maturation of DCs, immature mdDCs were stimulated with lipopolysaccharide $(100 \mathrm{ng} / \mathrm{ml}$; Sigma-Aldrich) following priming with interferon- $\gamma$ (EMD Millipore, Billerica, MA, USA) for $3 \mathrm{~h}$. MDA-MB-231 tumor-associated mdDCs (MDA-TADCs), BBP-MDA-TADCs, DBP-MDA-TADCs or DEHP-MDA-TADCs were generated by culturing $\mathrm{CD} 14^{+}$monocytes in $\alpha$-MEM medium containing FBS, IL4 and GM-CSF in 20\% MDA-CM, BBP-MDA-CM, DBP-MDA-CM or DEHP-MDA-CM, and subsequently stimulated as aforementioned. Following washing, the supernatants were collected and identified as MDA-MB-231-TADC-CM, BBP-MDA-TADC-CM, DBP-MDA-TADC-CM or DEHP-MDA-TADC-CM (Fig. 1B). The Institutional Review Board (IRB) of Kaohsiung Medical University Hospital (Kaohsiung, Taiwan) approved the present study protocol and all participants provided written informed consent in accordance with the Declaration of Helsinki (IRB numbers: KMUH-IRB-990174 and KMUH-IRB-20120362).

Reverse transcription-quantitative polymerase chain reaction (RT-qPCR). TRIzol reagent (Invitrogen; Thermo Fisher Scientific, Inc.) was utilized for RNA isolation, while complementary (c)DNA was prepared using an oligo(dT) primer and reverse transcriptase (Takara Bio, Inc., Otsu, Japan) following standard protocols (29). RT-qPCR was performed using SYBR Green on the ABI 7500 Real-Time PCR System (Applied Biosystems; Thermo Fisher Scientific, Inc.). Each PCR mixture contained $200 \mathrm{nM}$ of each primer, $10 \mu \mathrm{l}$ of $2 \mathrm{X}$ SYBR Green PCR Master Mix (Applied Biosystems; Thermo Fisher Scientific, Inc.), and $5 \mu \mathrm{l}$ of cDNA and RNase-free water, in a total volume of $20 \mu \mathrm{l}$. The RT-qPCR was performed with a denaturation step at $95^{\circ} \mathrm{C}$ for $10 \mathrm{~min}$, then for 40 cycles at $95^{\circ} \mathrm{C}$ for $15 \mathrm{sec}$ and $60^{\circ} \mathrm{C}$ for $1 \mathrm{~min}$. All PCRs were performed in triplicate and normalized to internal control glyceraldehyde-3-phosphate dehydrogenase mRNA. The relative expression level was presented using the $2^{-\Delta \Delta \mathrm{Cq}}$ method. The primer sequences of target genes in the present study were as follows: RANTES, F 5'-cgctgtcat cctcattgcta-3' and R 5'-acacacttggcggttctttc-3'; and GAPDH F 5'-GAGTCAACGGATTTGGTCGT-3' and R 5'-TTGATT TTGGAGGGATCTCG-3'.

Enzyme-linked immunosorbent assay (ELISA). RANTES levels were determined using an ELISA-based kit (R\&D Systems Europe Ltd., Abingdon, UK). ELISA was performed according to the manufacturer's protocols. Depletion of RANTES from various CMs was performed using a mouse monoclonal anti-RANTES antibodies $(2 \mu \mathrm{g} / \mathrm{ml}$; Abcam, Cmabridge, UK) and Sepharose ${ }^{\mathrm{TM}}$ Protein A/G beads (PAG50-00-0002, Rockland Immunochemicals Inc., Gilbertsville, PA, USA) following standard immunoprecipitation techniques (30). Cytokine depletion was additionally confirmed using an ELISA-based kit.

Cell proliferation. The cells were plated in 96-well culture plates. Following $24 \mathrm{~h}$ of incubation, the cells were treated with vehicle mdDC-CM or specific CM for $72 \mathrm{~h}$. At the conclusion of the assay period, cell proliferation was measured using a water-soluble tetrazolium salts (WST)-1 assay. Cell proliferation was determined using Premixed WST-1 Cell Proliferation Reagent (Clontech Laboratories, Inc., Mountainview, CA, USA) in accordance with the manufacturer's protocols.

Cell migration and invasion assay. Cell migration and invasion assays were performed using QCM $^{\mathrm{TM}}$ 24-well Cell Migration and Invasion Assay kits (EMD Millipore). Briefly, the cells were seeded into the migration chamber and mdDC-CM or various $\mathrm{CM}$ were added to the lower wells for $24 \mathrm{~h}$ as a chemoattractant. At the conclusion of the treatment, the cells were stained using CyQuant GR dye (cat no. 90131) in cell lysis buffer for $15 \mathrm{~min}$ at room temperature. The fluorescence of the migrated and invaded cells was subsequently measured using a fluorescence plate reader at excitation/emission wavelengths of 485/520 nm.

Statistical analysis. Data are expressed as the mean \pm standard deviation. Statistical comparisons of the results were 
A

B

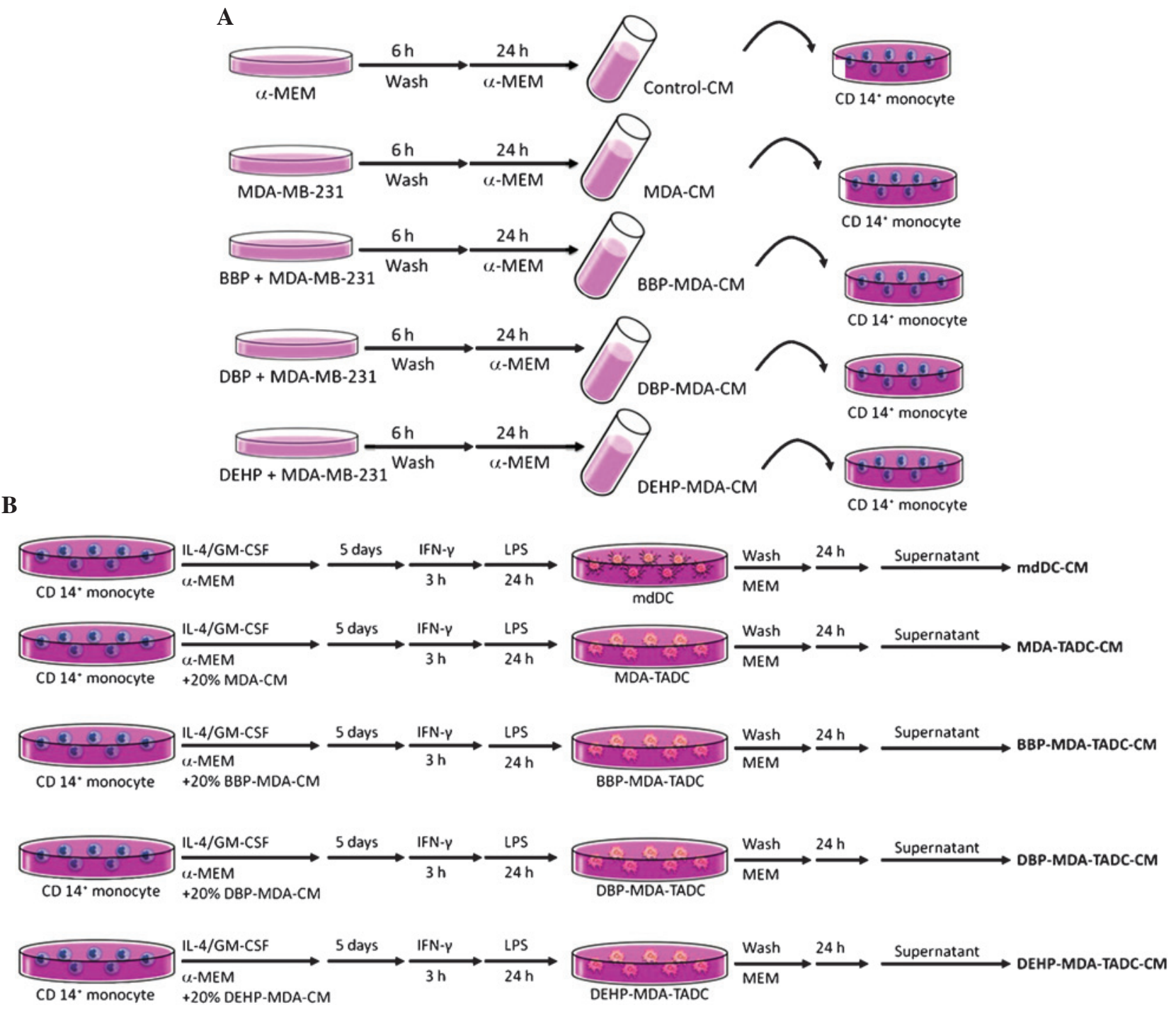

Figure 1. Flow chart of the production of various CM. (A) Flow chart of the production of control-CM, MDA-CM, BBP-MDA-CM, DBP-MDA-CM and DEHP-MDA-CM. (B) Flow chart of the production of mdDC-CM, MDA-TADC-CM, BBP-MDA-TADC-CM, DBP-MDA-TADC-CM and DEHP-MDA-TADC-CM. CM, conditioned media; MDA, MDA-MB-231 cells; BBP, butyl benzyl phthalate; DBP, di- $n$-butyl phthalate; DEHP, di-2-ethylhexyl phthalate; mdDC, monocyte-derived dendritic cells; TADC, tumor-associated mdDC; MEM, minimum essential medium; IL, interleukin; GM-CSF, granulocyte-macrophage colony-stimulating factor; IFN, interferon; LPS, lipopolysaccharide; CD, cluster of differentiation.

performed using an analysis of variance. Significant differences between the means of the test groups were analyzed using Student's t-test. $\mathrm{P}<0.05$ was considered to indicate a statistically significant difference.

\section{Results}

Breast cancer cells, following exposure to phthalate esters, affect $m d D C s$ and contribute to breast cancer progression by enhancing cancer cell proliferation, migration and invasion. In order to understand whether phthalate esters exacerbate cancer progression in the breast cancer tumor microenvironment, the effects of BBP-MDA-TADC-CM, DBP-MDA-TADC-CM and DEHP-MDA-TADC-CM on breast cancer cell proliferation, migration and invasion were investigated. MDA-TADC-CM $(20 \%)$ increased breast cancer cell proliferation $(\mathrm{P}=0.01)$, and this stimulatory effect was additionally enhanced when MDA-MB-231 cells were pretreated with BBP, DBP or DEHP (Fig. 2A) (P=0.001,0.0001 and 0.0007 for BBP, DBP and DEHP, respectively). In addition, MDA-TADC-CM (20\%) induced breast cancer cell migration and invasion $(\mathrm{P}=0.002$ and 0.008 for migration and invasion analysis), and this reinforceable effect was worsened when breast cancer cells were cultured with BBP-MDA-TADC-CM (20\%), DBP-MDA-TADC-CM (20\%) or DEHP-MDA-TADC-CM (20\%) (Fig. 2B and C).

RANTES has a significant role in TADC-mediated cancer progression. In order to determine the primary factors contributing to MDA-TADC, BBP-MDA-TADC, DBP-MDA-TADC and DEHP-MDA-TADC-mediated breast cancer progression, RT-qPCR analysis revealed that RANTES mRNA levels were increased by 6-, 12-, 17- and 11-fold in MDA-TADCs, BBP-MDA-TADCs, DBP-MDA-TADCs and DEHP-MDA-TADCs, respectively (Fig. 3A). As demonstrated by ELISA, the protein levels of RANTES were enhanced in MDA-TADC-CM $(\mathrm{P}=0.01)$, BBP-MDA-TADC-CM ( $\mathrm{P}=0.005)$, DBP-MDA-TADC-CM $(\mathrm{P}=0.002)$ and DEHP-MDA-TADC-CM (Fig. 3B) 

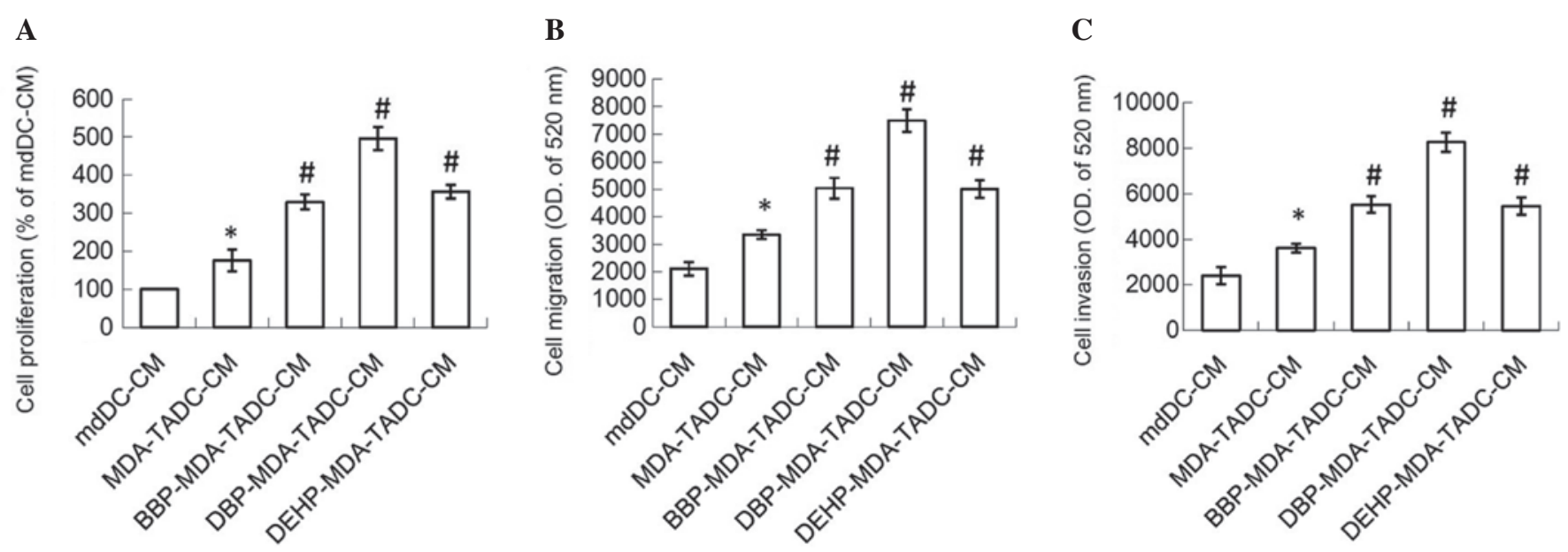

Figure 2. CM of phthalate ester-treated MDA-MB-231 cells cause mdDCs to increase breast cancer cell proliferation, migration and invasion. BBP-, DBP- and DEHP-TADC-CM increased breast cancer cell (A) proliferation, (B) migration and (C) invasion. Each value is presented as the mean \pm standard deviation of three independent experiments. ${ }^{*} \mathrm{P}<0.05$ vs. mdDC-CM treatment. ${ }^{*} \mathrm{P}<0.05$ vs. MDA-TADC-CM treatment. CM, conditioned media; mdDCs, monocyte-derived dendritic cells; BBP, butyl benzyl phthalate; DBP, di- $n$-butyl phthalate; DEHP, di-2-ethylhexyl phthalate; TADC, tumor-associated mdDC; MEM, minimum essential medium; OD, optical density.

$(\mathrm{P}=0.003)$. The effect of DBP-MDA-TADC-CM on the induction of breast cancer cell proliferation, migration and invasion, and the induction of RANTES was greater than that of BBP-MDA-TADC-CM or DEHP-MDA-TADC-CM (Figs. 2 and 3). As DBP demonstrated the greatest effect in these circumstances, it was selected as the model for investigation of the detailed effects of phthalate ester-associated cancer aggravation in the breast cancer tumor microenvironment. In order to understand its role, RANTES was depleted in MDA-TADC-CM and DBP-MDA-TADC-CM. The effect of MDA-TADC-CM and DBP-MDA-TADC-CM on cancer progression was subsequently assessed. The successful depletion of RANTES from MDA-TADC-CM and DBP-MDA-TADC-CM was confirmed using RANTES ELISA kits (data not shown). As demonstrated in Fig. 4, RANTES depletion inhibited the stimulatory effects of MDA-TADC-CM on breast cancer cell proliferation, migration and invasion $(\mathrm{P}=0.00002$ for cell proliferation, $\mathrm{P}<0.0001$ for migration and invasion), as well as blocking the intensified stimulatory effects of DBP-MDA-TADC-CM on breast cancer cell proliferation, migration and invasion.

Didymin suppresses DBP-MDA-TADC-mediated breast cancer aggravation. The tumor microenvironment has a significant role in the development of chemoresistance to anticancer drugs and tumor progression (7). TADC has been demonstrated to promote the progression of cancer by modulating a number of components in the cancer niche, thereby creating a supportive and permissive microenvironment for tumor survival, proliferation and metastasis $(11,31)$. As phthalate esters stimulate the ability of breast cancer cells to affect mdDCs and thereby intensified breast cancer cell proliferation, migration and invasion, the search for a possible antidote in the fight against phthalate esters-induced cancer aggravation in the breast cancer tumor microenvironment has become a matter of importance. The present study therefore assessed the effect of didymin, a dietary flavonoid glycoside derived from citrus fruits, on DBP-induced cancer progression.
As shown in Fig. 5A, the MDA-TADC-CM-induced breast cancer cell proliferation effect was inhibited when cells were pretreated with didymin, and DBP-MDA-TADC-CM-induced breast cancer cell proliferation was additionally reversed when the cells were pretreated with didymin. Similarly, enhancement of breast cancer cell migration and invasion triggered by DBP-MDA-TADC-CM was abrogated upon didymin pretreatment (Fig. 5B and C).

\section{Discussion}

To the best of our knowledge, the present study is the first to evaluate the interaction between mdDCs and breast cancer cells following exposure to phthalate esters. BBP, DBP and DEHP stimulated the breast cancer cells and subsequently caused the mdDCs to secrete RANTES, which enhanced the proliferation, migration and invasion of the human breast cancer cells. To the best of our knowledge, the present study is additionally the first to investigate the effects of didymin in interfering with phthalate ester-mediated breast cancer aggravation in the breast cancer tumor microenvironment. The results of the present study suggested that didymin was capable of preventing phthalate ester-associated breast cancer progression (Fig. 6).

Phthalates, including BBP, DBP and DEHP, are widely utilized in food wraps and cosmetic products (1-3). Individuals are exposed to phthalates throughout their entire lives via ingestion, inhalation and dermal exposure $(1,2)$. Several phthalates have been demonstrated to promote breast cancer development by increasing cell proliferation and migration (32-34). In a previous study, we demonstrated that phthalate esters were able to induce breast cancer bone metastasis by targeting parathyroid hormone-related protein (35). The present study demonstrated that phthalate esters BBP, DBP and DEHP were able to induce breast cancer cells to affect mdDCs, thereby intensifying breast cancer cell proliferation, migration and invasion. The results of the present study suggested that phthalate esters may increase cancer progression in the breast cancer tumor microenvironment. 

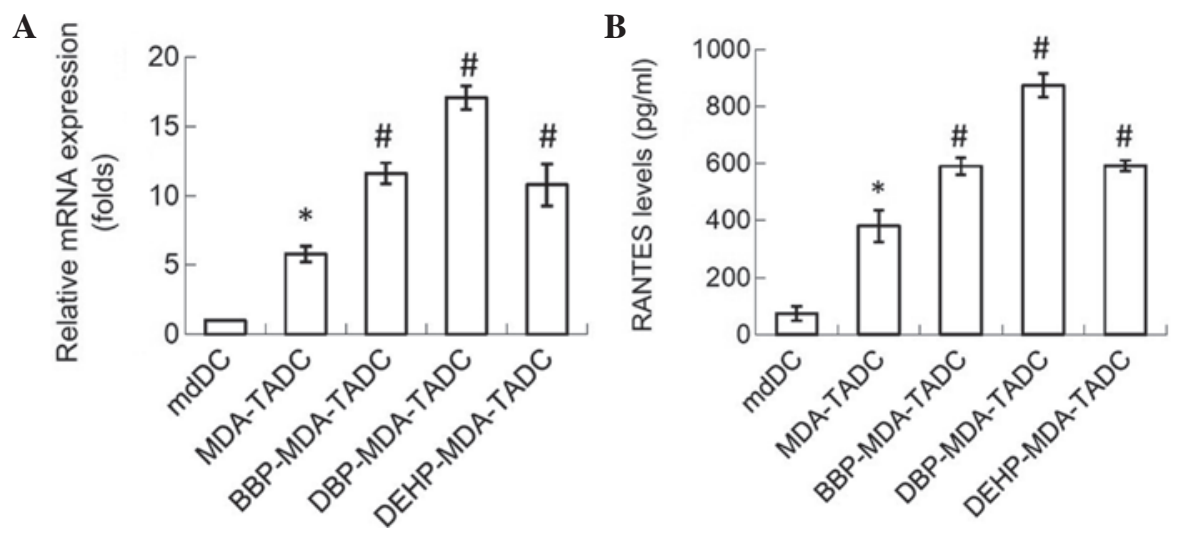

Figure 3. MDA-CM, BBP-MDA-CM, DBP-MDA-CM and DEHP-MDA-CM increase the expression of RANTES in mdDCs. (A) MDA-CM, BBP-MDA-CM, DBP-MDA-CM and DEHP-MDA-CM increased the levels of RANTES, as assessed by reverse transcription-polymerase chain reaction. (B) MDA-CM, BBP-MDA-CM, DBP-MDA-CM and DEHP-MDA-CM increased the RANTES protein levels, as detected by enzyme-linked immunosorbent assay. Each value is presented as the mean \pm standard deviation of three independent experiments. ${ }^{*} \mathrm{P}<0.05$ vs. mdDC treatment. ${ }^{*} \mathrm{P}<0.05$ vs. MDA-TADC treatment. MDA, MDA-MB-231 cells; CM, conditioned media; BBP, butyl benzyl phthalate; DBP, di- $n$-butyl phthalate; DEHP, di-2-ethylhexyl phthalate; RANTES; regulated upon activation, normal T-cell expressed, and secreted; mdDCs, monocyte-derived dendritic cells.

A

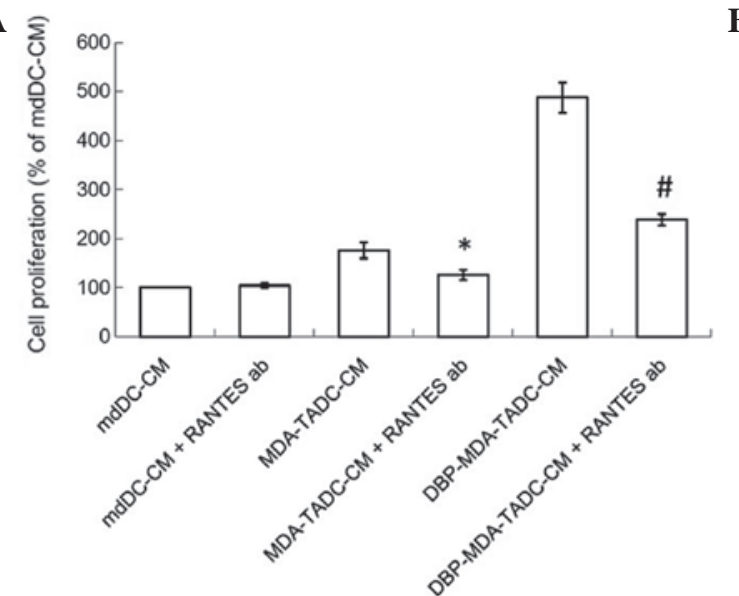

B

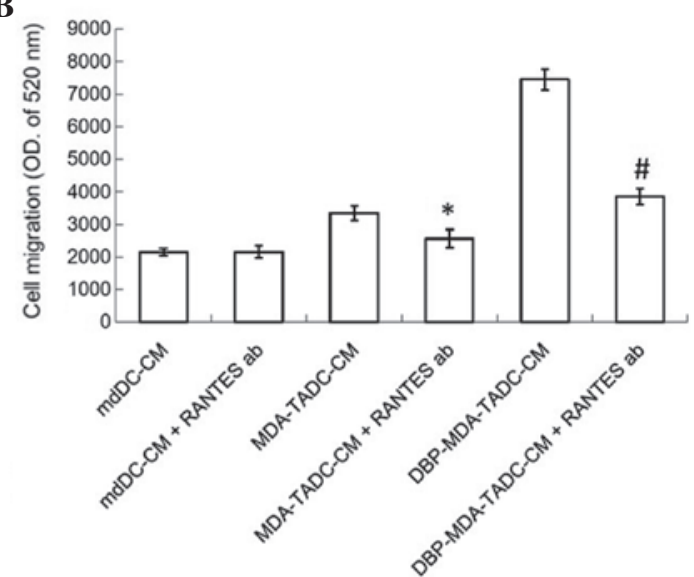

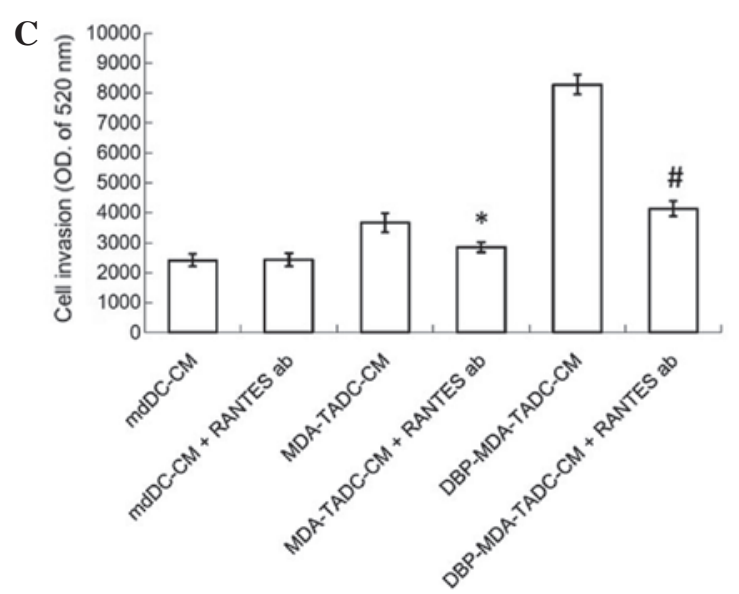

Figure 4. RANTES is a significant factor for MDA-TADC-CM and DBP-MDA-TADC-CM in increasing breast cancer cell proliferation and migration (A) The proliferation of MDA-MB-231 cells observed in RANTES-depleted MDA-TADC-CM or DBP-MDA-TADC-CM. (B) Migration and (C) invasion of MDA-MB-231 cells present in RANTES-depleted MDA-TADC-CM or DBP-MDA-TADC-CM. Each value is presented as the mean \pm standard deviation of three independent experiments. "P $<0.05$ vs. MDA-TADC-CM treatment. ${ }^{*} \mathrm{P}<0.05$ vs. DBP-MDA-TADC-CM treatment. RANTES; regulated upon activation, normal T-cell expressed, and secreted; MDA, MDA-MB-231 cells; mdDCs, monocyte-derived dendritic cells; TADC, tumor-associated mdDC; CM, conditioned media; DBP, di- $n$-butyl phthalate; OD, optical density; ab, antibody.

The tumor microenvironment has been observed to affect cancer progression and generation. The cells surrounding a tumor provide essential supportive factors that promote progression (36-39). RANTES has been observed to be a significant contributor to various chronic inflammatory diseases and malignancies via the recruitment of inflammatory 


\section{A}

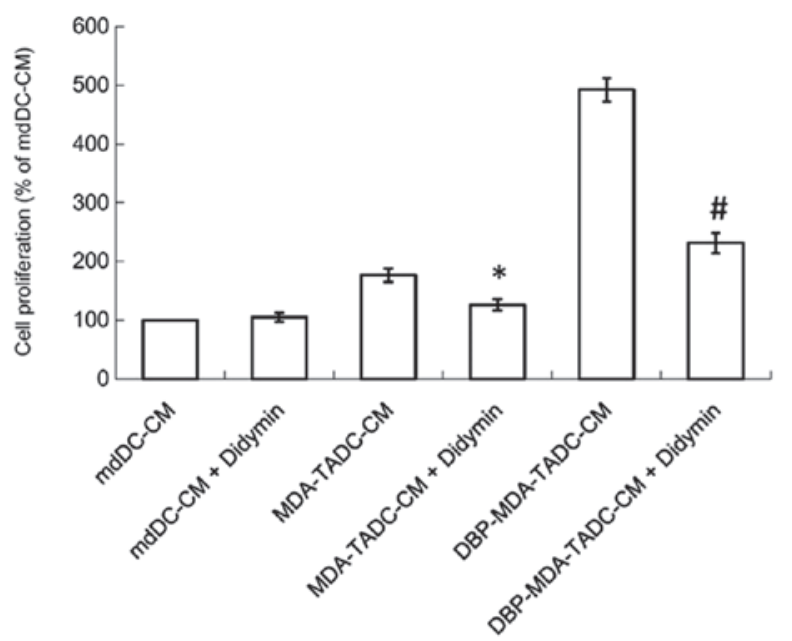

C
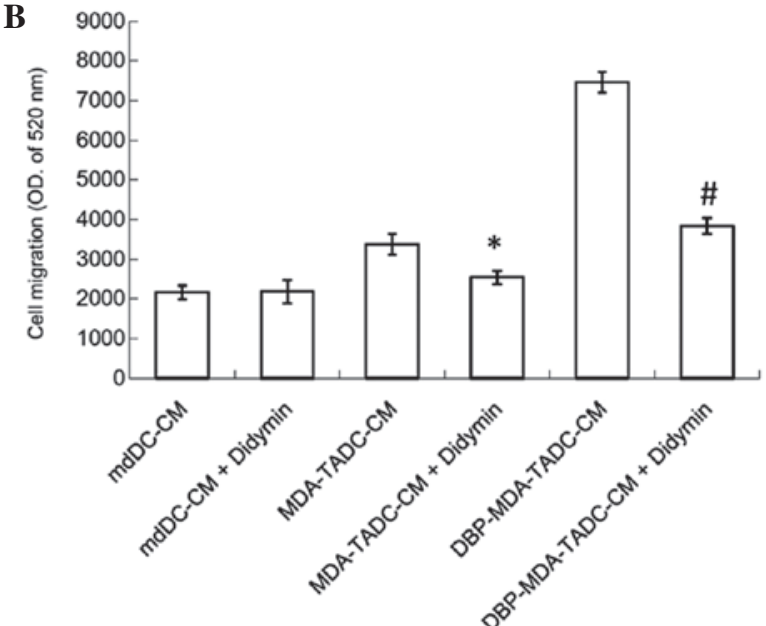

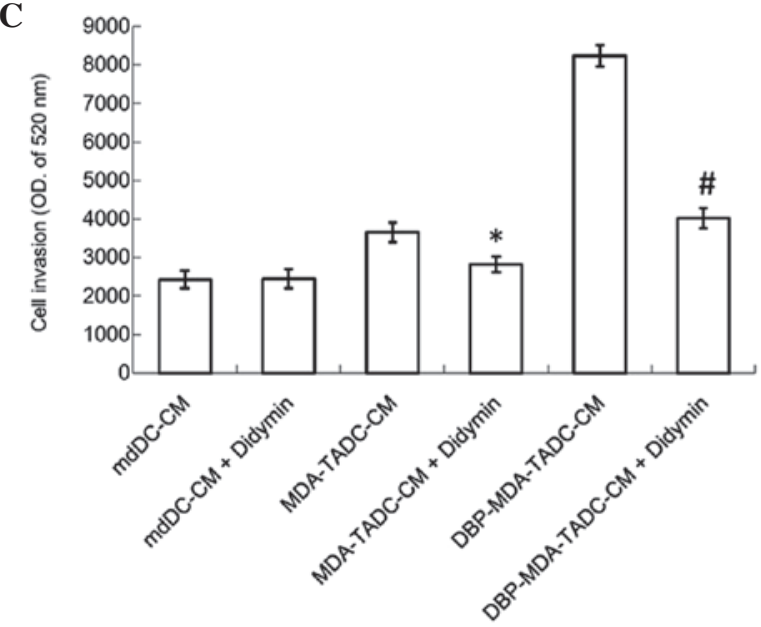

Figure 5. Didymin suppresses DBP-mediated cancer aggravation in the breast cancer tumor microenvironment. Didymin reversed (A) proliferation, (B) migration and (C) invasion of MDA-MB-231 breast cancer cells exposed in culture to MDA-TADC-CM or DBP-MDA-TADC-CM. Each value is presented as the mean \pm standard deviation of three independent experiments. "P<0.05 vs. MDA-TADC-CM treatment. ${ }^{*} \mathrm{P}<0.05$ vs. DBP-MDA-TADC-CM treatment. DBP, di- $n$-butyl phthalate; mdDCs, monocyte-derived dendritic cells; TADC, tumor-associated mdDC; CM, conditioned media; OD, optical density.

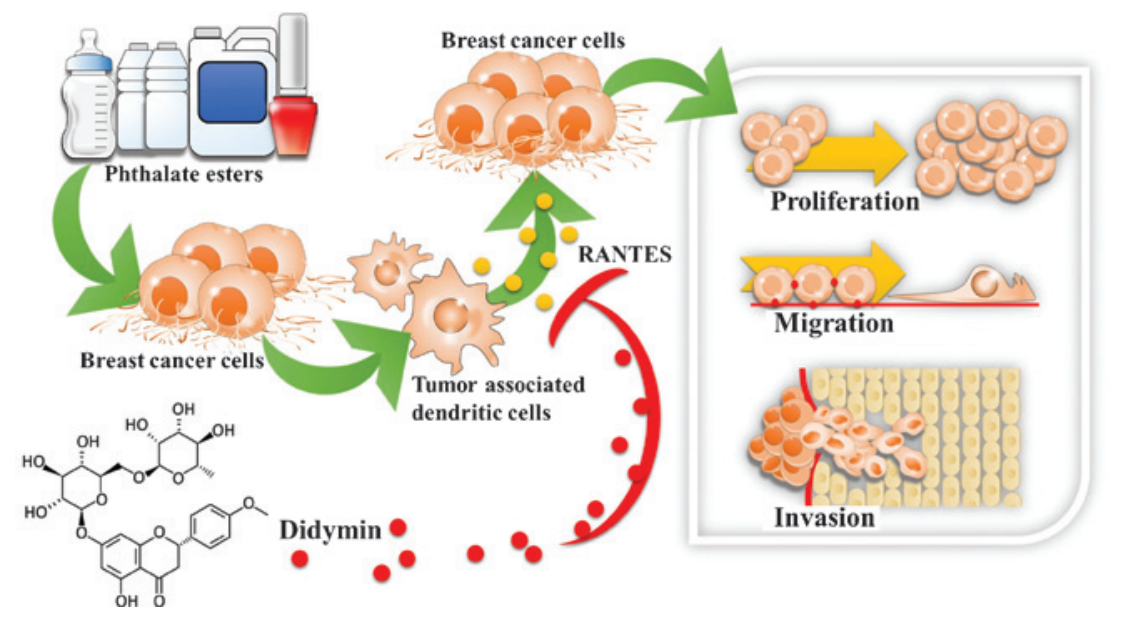

Figure 6. Didymin reverses phthalate ester-associated breast cancer aggravation in the breast cancer tumor microenvironment. The present study demonstrated two novel findings: i) RANTES, produced by breast tumor-associated monocyte-derived dendritic cells following breast cancer cells' exposure to phthalate esters, contributed to cancer progression by enhancing cancer cell proliferation, migration and invasion; and ii) didymin, a dietary flavonoid glycoside present in citrus fruits, reversed phthalate ester-mediated breast cancer aggravation.

cells $(15,40,41)$. RANTES has additionally been reported to be overexpressed in certain cancers and is involved in critical steps of cancer spread, including proliferation, migration, invasion, angiogenesis and metastatic colonization following activation (42-45). Furthermore, RANTES has been associated with resistance to conventional chemotherapeutic drugs, including 
cisplatin and tamoxifen (46). The present study revealed that the phthalate esters BBP, DBP and DEHP were able to cause breast cancer cells to affect mdDCs to produce the inflammatory cytokine RANTES, which subsequently enhanced breast cancer cell proliferation, migration and invasion. Depletion of RANTES reversed the effects of MDA-TADC-CM- and DBP-MDA-TADC-CM-mediated breast cancer cell proliferation, migration and invasion. Elimination of all phthalate exposure may not be possible, as phthalate esters are extensively used in modern life $(1,2)$. It is therefore important that strategies are developed for the prevention of breast cancer progression in the breast cancer tumor microenvironment. The results of the present study revealed that didymin, a dietary flavonoid glycoside obtained from citrus fruits, is able to reverse the negative actions of DBP-stimulated breast cancer cells, which cause mdDCs to enhance the proliferation, migration and invasion of human breast cancer cells.

In conclusion, to the best of our knowledge, the present study is the first to investigate the interaction between mdDCs and breast cancer cells following exposure to phthalate esters. These esters, BBP, DBP and DEHP, were used to stimulate human breast cancer MDA-MB-231 cells to mediate RANTES upregulation in mdDCs, thereby inducing breast cancer cell proliferation, migration and invasion. To the best of our knowledge, this is the first study to provide evidence that didymin, a dietary flavonoid glycoside present in citrus fruits, has potential for the prevention of phthalate ester-associated breast cancer progression in the breast cancer tumor microenvironment. Therefore, the present study suggested that didymin may be capable of preventing phthalate ester-associated cancer aggravation.

\section{Acknowledgements}

The present study was supported by grants from the National Science Council of Taiwan (nos. NSC 102-2628-B-037-002-MY3, NSC 102-2632-B-037-001-MY3, and NSC 102-2314-B-037-035-MY3), the Kaohsiung Medical University 'Aim for the Top 500 Universities Grant' (no. KMU-DT103008), the Kaohsiung Medical University 'Aim for the Top Universities Grant' (no. KMU-TP103A15), the Kaohsiung Medical University Hospital Research Foundation (no. KMUH103-3M53) and the Excellence for Cancer Research Center Grant, the Ministry of Health and Welfare, Executive Yuan (Taipei, Taiwan; no. MOHW 103-TD-B-111-05).

\section{References}

1. Martino-Andrade AJ and Chahoud I: Reproductive toxicity of phthalate esters. Mol Nutr Food Res 54: 148-157, 2010.

2. Ge RS, Chen GR, Tanrikut C and Hardy MP: Phthalate ester toxicity in Leydig cells: Developmental timing and dosage considerations. Reprod Toxicol 23: 366-373, 2007.

3. López-Carrillo L, Hernández-Ramírez RU, Calafat AM, Torres-Sánchez L, Galván-Portillo M, Needham LL, Ruiz-Ramos R and Cebrián ME: Exposure to phthalates and breast cancer risk in northern Mexico. Environ Health Perspect 118: 539-544, 2010.

4. Aschengrau A, Coogan PF, Quinn M and Cashins LJ: Occupational exposure to estrogenic chemicals and the occurrence of breast cancer: An exploratory analysis. Am J Ind Med 34: 6-14, 1998.

5. Carran M and Shaw IC: New Zealand Malayan war veterans' exposure to dibutylphthalate is associated with an increased incidence of cryptorchidism, hypospadias and breast cancer in their children. N Z Med J 125: 52-63, 2012.
6. Sprague BL, Trentham-Dietz A, Hedman CJ, Wang J, Hemming JD, Hampton JM, Buist DS, Aiello Bowles EJ, Sisney GS and Burnside ES: Circulating serum xenoestrogens and mammographic breast density. Breast Cancer Res 15: R45, 2013.

7. Katoh H, Wang D, Daikoku T, Sun H, Dey SK and Dubois RN: CXCR2-expressing myeloid-derived suppressor cells are essential to promote colitis-associated tumorigenesis. Cancer Cell 24: 631-644, 2013.

8. Peltekova VD, Lemire M, Qazi AM, Zaidi SH, Trinh QM, Bielecki R, Rogers M, Hodgson L, Wang M, D'Souza DJ, et al: Identification of genes expressed by immune cells of the colon that are regulated by colorectal cancer-associated variants. Int J Cancer 134: 2330-2341, 2014.

9. Gajewski TF, Schreiber H and Fu YX: Innate and adaptive immune cells in the tumor microenvironment. Nat Immunol 14: 1014-1022, 2013.

10. Hsu YL, Huang MS, Cheng DE, Hung JY, Yang CJ, Chou SH and Kuo PL: Lung tumor-associated dendritic cell-derived amphiregulin increased cancer progression. J Immunol 187: 1733-1744, 2011.

11. Kuo CH, Chen KF, Chou SH, Huang YF, Wu CY, Cheng DE, Chen YW, Yang CJ, Hung JY and Huang MS: Lung tumor-associated dendritic cell-derived resistin promoted cancer progression by increasing Wolf-Hirschhorn syndrome candidate 1/Twist pathway. Carcinogenesis 34: 2600-2609, 2013.

12. Gonzalez RM, Daly DS, Tan R, Marks JR and Zangar RC: Plasma biomarker profiles differ depending on breast cancer subtype but RANTES is consistently increased. Cancer Epidemiol Biomarkers Prev 20:1543-1551, 2011.

13. Velasco-Velázquez M and Pestell RG: The CCL5/CCR5 axis promotes metastasis in basal breast cancer. Oncoimmunology 2: e23660, 2013.

14. Lv D, Zhang Y, Kim HJ, Zhang L and Ma X: CCL5 as a potential immunotherapeutic target in triple-negative breast cancer. Cell Mol Immunol 10: 303-310, 2013.

15. Zhang Y, Lv D, Kim HJ, Kurt RA, Bu W, Li Y and Ma X: A novel role of hematopoietic CCL5 in promoting triple-negative mammary tumor progression by regulating generation of myeloid-derived suppressor cells. Cell Res 23: 394-408, 2013.

16. Ash SA, Valchev GI, Looney M, Ni Mhathuna A, Crowley PD, Gallagher HC and Buggy DJ: Xenon decreases cell migration and secretion of a pro-angiogenesis factor in breast adenocarcinoma cells: Comparison with sevoflurane. Br J Anaesth 113 (Suppl 1): i14-i21, 2014.

17. Takemura H, Sakakibara H, Yamazaki S and Shimoi K: Breast cancer and flavonoids - a role in prevention. Curr Pharm Des 19: 6125-6132, 2013.

18. Donaldson MS: Nutrition and cancer: A review of the evidence for an anti-cancer diet. Nutr J 3: 19, 2004.

19. Stoner GD and Mukhtar H: Polyphenols as cancer chemopreventive agents. J Cell Biochem Suppl 22: 169-180, 1995.

20. Yang CS, Landau JM, Huang MT and Newmark HL: Inhibition of carcinogenesis by dietary polyphenolic compounds. Annu Rev Nutr 21: 381-406, 2001.

21. Aggarwal BB and Shishodia S: Molecular targets of dietary agents for prevention and therapy of cancer. Biochem Pharmacol 71: 1397-1421, 2006.

22. Ramos AM and Aller P: Quercetin decreases intracellular GSH content and potentiates the apoptotic action of the antileukemic drug arsenic trioxide in human leukemia cell lines. Biochem Pharmacol 75: 1912-1923, 2008.

23. Calabrò ML, Galtieri V, Cutroneo P, Tommasini S, Ficarra P and Ficarra R: Study of the extraction procedure by experimental design and validation of a LC method for determination of flavonoids in Citrus bergamia juice. J Pharm Biomed Anal 35: 349-363, 2004

24. Finotti E and Di Majo D: Influence of solvents on the antioxidant property of flavonoids. Nahrung 47: 186-187, 2003.

25. Mouly P, Gaydou EM and Auffray A: Simultaneous separation of flavanone glycosides and polymethoxylated flavones in citrus juices using liquid chromatography. J Chromatogr A 800: 171-179, 1998.

26. Ross SA, Ziska DS, Zhao K and ElSohly MA: Variance of common flavonoids by brand of grapefruit juice. Fitoterapia 71: 154-161, 2000.

27. Hung JY, Hsu YL, Ko YC, Tsai YM, Yang CJ, Huang MS and Kuo PL: Didymin, a dietary flavonoid glycoside from citrus fruits, induces Fas-mediated apoptotic pathway in human non-small-cell lung cancer cells in vitro and in vivo. Lung Cancer 68: 366-374, 2010. 
28. Singhal J, Nagaprashantha LD, Vatsyayan R, Ashutosh, Awasthi S and Singhal SS: Didymin induces apoptosis by inhibiting N-Myc and upregulating RKIP in neuroblastoma. Cancer Prev Res (Phila) 5: 473-483, 2012.

29. Zeng F, Xie L, Pang X, Liu W, Nie Q and Zhang X: Complementary eoxyribonucleic acid cloning of avian G0/G1 switch gene 2, and its expression and association with production traits in chicken. Poult Sci 90: 1548-1554, 2011.

30. Bharadwaj U, Li M, Zhang R, Chen C and Yao Q: Elevated interleukin- 6 and G-CSF in human pancreatic cancer cell conditioned medium suppress dendritic cell differentiation and activation. Cancer Res 67: 5479-5488, 2007.

31. Watkins SK, Zhu Z, Riboldi E, Shafer-Weaver KA, Stagliano KE, Sklavos MM, Ambs S, Yagita $\mathrm{H}$ and Hurwitz AA: FOXO3 programs tumor-associated DCs to become tolerogenic in human and murine prostate cancer. J Clin Invest 121: 1361-1372, 2011.

32. Hsieh TH, Tsai CF, Hsu CY, Kuo PL, Hsi E, Suen JL, Hung CH, Lee JN, Chai CY, Wang SC and Tsai EM: n-Butyl benzyl phthalate promotes breast cancer progression by inducing expression of lymphoid enhancer factor 1. PLoS One 7: e42750, 2012.

33. Hsieh TH, Tsai CF, Hsu CY, Kuo PL, Lee JN, Chai CY, Wang SC and Tsai EM: Phthalates induce proliferation and invasiveness of estrogen receptor-negative breast cancer through the AhR/HDAC6/c-Myc signaling pathway. FASEB J 26: 778-787, 2012.

34. Chen FP and Chien MH: Lower concentrations of phthalates induce proliferation in human breast cancer cells. Climacteric 17: 377-384, 2014.

35. Hsu YL, Tsai EM, Hou MF, Wang TN, Hung JY and Kuo PL: Obtusifolin suppresses phthalate esters-induced breast cancer bone metastasis by targeting parathyroid hormone-related protein. J Agric Food Chem 62: 11933-11940, 2014.

36. Tsai MJ, Chang WA, Huang MS and Kuo PL: Tumor microenvironment: A new treatment target for cancer. ISRN Biochem 2014: 351959, 2014

37. Liu Y, Han ZP, Zhang SS, Jing YY, Bu XX, Wang CY, Sun K, Jiang GC, Zhao X, Li R, et al: Effects of inflammatory factors on mesenchymal stem cells and their role in the promotion of tumor angiogenesis in colon cancer. J Biol Chem 286: 25007-25015, 2011.

38. Luo YP, Zhou H, Krueger J, Kaplan C, Liao D, Markowitz D, Liu C, Chen T, Chuang TH, Xiang R and Reisfeld RA: The role of proto-oncogene Fra-1 in remodeling the tumor microenvironment in support of breast tumor cell invasion and progression. Oncogene 29: 662-673, 2010
39. Hsu YL, Hou MF, Kuo PL, Huang YF and Tsai EM: Breast tumor-associated osteoblast-derived CXCL5 increases cancer progression by ERK/MSK1/Elk-1/snail signaling pathway. Oncogene 32: 4436-4447, 2013.

40. Chang LY, Lin YC, Mahalingam J, Huang CT, Chen TW, Kang CW, Peng HM, Chu YY, Chiang JM, Dutta A, et al: Tumor-derived chemokine CCL5 enhances TGF- $\beta$-mediated killing of CD8(+) T cells in colon cancer by T-regulatory cells. Cancer Res 72: 1092-1102, 2012.

41. Soria G, Ofri-Shahak M,Haas I, Yaal-Hahoshen N,Leider-Trejo L, Leibovich-Rivkin T, Weitzenfeld P, Meshel T, Shabtai E, Gutman $\mathrm{M}$ and Ben-Baruch A: Inflammatory mediators in breast cancer: Coordinated expression of TNF $\alpha$ \& IL-1 $\beta$ with CCL2 \& CCL5 and effects on epithelial-to-mesenchymal transition. BMC Cancer 11: 130, 2011.

42. Borczuk AC,Papanikolaou N,Toonkel RL, Sole M, GorensteinLA, Ginsburg ME, Sonett JR, Friedman RA and Powell CA: Lung adenocarcinoma invasion in TGFbetaRII-deficient cells is mediated by CCL5/RANTES. Oncogene 27: 557-564, 2008.

43. Mi Z, Bhattacharya SD, Kim VM, Guo H, Talbot LJ and Kuo PC: Osteopontin promotes CCL5-mesenchymal stromal cell-mediated breast cancer metastasis. Carcinogenesis 32: 477-487, 2011.

44. Trellakis S, Bruderek K, Dumitru CA, Gholaman H, Gu X, Bankfalvi A, Scherag A, Hütte J, Dominas N, Lehnerdt GF, et al: Polymorphonuclear granulocytes in human head and neck cancer: Enhanced inflammatory activity, modulation by cancer cells and expansion in advanced disease. Int J Cancer 129: 2183-2193, 2011

45. Jiao X, Katiyar S, Willmarth NE, Liu M, Ma X, Flomenberg N, Lisanti MP and Pestell RG: c-Jun induces mammary epithelial cellular invasion and breast cancer stem cell expansion. J Biol Chem 285: 8218-8226, 2010.

46. Yi EH, Lee CS, Lee JK, Lee YJ, Shin MK, Cho CH, Kang KW, Lee JW, Han W, Noh DY, et al: STAT3-RANTES autocrine signaling is essential for tamoxifen resistance in human breast cancer cells. Mol Cancer Res 11: 31-42, 2013. 